\title{
Colon cancer with colosplenic fistula
}

\begin{abstract}
A previously healthy woman presented with gram-negative rod sepsis and profound anemia in the context of functional decline over a period of months. Abdominal cross sectional imaging was concerning for widely metastatic colon cancer as well as gas in the spleen, suggestive of a colosplenic fistula. Colonoscopy was deferred in favor of diagnostic laparoscopy for tissue diagnosis. Shallow colonic biopsy was highly suggestive of adenocarcinoma. She underwent a loop ileostomy and loop transverse colostomy in hopes of facilitating healing of the fistula so that she could receive chemotherapy. Splenic flexure resection and splenectomy were deferred due to high operative risk. In light of postoperative complications, she ultimately elected for hospice care.
\end{abstract}

Keywords: Colon cancer; Fistula; Colosplenic fistula; Enteric fistula; Colorectal surgery

\author{
Volume 7 Issue 6 - 2017
}

\begin{abstract}
Snell David B,' Desai Amit P,' Wan David W'
'Department of Medicine, New York-Presbyterian/Weill Cornell Medical Center, USA

${ }^{2}$ Department of Gastroenterology and Hepatology, New YorkPresbyterian/Weill Cornell Medical Center, USA
\end{abstract}

Correspondence: David B Snell, Department of Medicine, 505 East 70th Street, HT-4, New York, NY, USA, Tel 212-746-3587, Email das9206@nyp.org

Received: July 26, 2017| Published: October 30, 2017
Abbreviations: IV, intravenous; E. coli, escherichia coli; CT, computed tomography

\section{Introduction}

Enteric fistulas commonly arise from inflammatory, infectious, or neoplastic processes. Surgical guidelines for enteric fistulas involve resection of the involved intestinal segment and recipient organ. Colosplenic fistulas are rare and surgical management guidelines are not well-established. Colosplenic fistulas are also thought to present challenges in endoscopic evaluation of the colon given the potential for splenic rupture with insufflation.

\section{Case presentation}

A 52-year-old woman with no medical history presented with 1-2 days of altered mental status in the setting of progressive functional decline over several months. She was febrile and tachycardic with a hemoglobin of 4.6, leukocytosis, lactic acidosis, and acute kidney injury. Initial resuscitation included IV fluids, blood transfusions, and broad-spectrum antibiotics. Subsequent blood cultures grew E. coli and Bacteroides. Abdominal CT was remarkable for gas in the spleen and splenic flexure wall thickening, concerning for colon cancer with invasion into the spleen and colosplenic fistula. Imaging revealed widely metastatic disease involving the liver, lungs, and spine. Colonoscopy was avoided because of the potential for splenic rupture on insufflation in the presence of the fistula. The initial plan for splenic flexure resection and splenectomy was rejected due to high operative risk. Instead, the patient underwent laparoscopic loop ileostomy and loop transverse colostomy to facilitate healing of the colosplenic fistula in hopes that chemotherapy could be initiated more quickly. Diagnostic laparoscopy demonstrated colonic perforation along with peritoneal metastases. Shallow biopsy of a non-obstructing mass in the splenic flexure resulted as villous adenoma with high-grade dysplasia. Post-operative imaging was significant for a pelvic fluid collection and splenic hypodensity concerning for metastasis or abscess. The patient was not a candidate for further surgical intervention and was transitioned to comfort measures only.

\section{Discussion}

Colosplenic fistulas are extremely rare with only a few case reports noted in the literature. Like other enteric fistulas, they arise from infectious, neoplastic, and inflammatory processes such as diverticulitis, colorectal cancer, lymphoma, pancreatitis, splenic abscesses and, most commonly, fistulizing Crohn's Disease. ${ }^{1,2}$ Colosplenic fistulas require cross-sectional imaging for diagnosis and further management. ${ }^{3}$ There are no well-defined guidelines for the management of colosplenic fistulas specifically in the setting of colorectal cancer. Although colonoscopy for the diagnosis of colorectal cancer has been performed in a few cases without incident, the use of colonoscopy in this setting is generally not recommended. ${ }^{4}$ In our case, colonoscopy was avoided due to the potential risk for splenic rupture on insufflation. Splenic injury after colonoscopy is very rare, with an incidence of 1-21 cases per 100,000. ${ }^{5}$ The majority of cases result from trauma, but infectious and neoplastic causes constitute the bulk of atraumatic causes. ${ }^{6}$ Therefore, in the setting of a colosplenic fistula, colonoscopy should be deferred in favor of a surgical biopsy for the diagnosis of colorectal cancer (Figures 1-3).

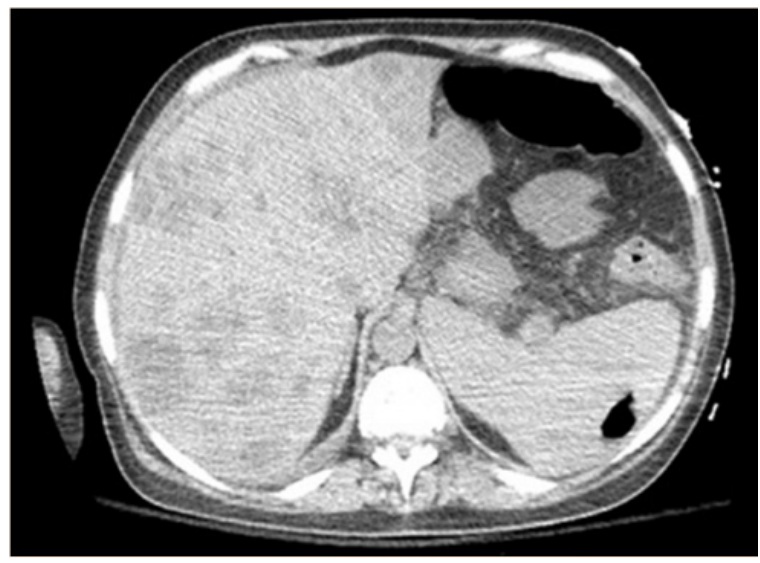

Figure I CT Abdomen/Pelvis (transverse plane) demonstrating gas collection along the posterolateral spleen measuring $4.3 \times 2.6 \times 2.1 \mathrm{~cm}$.

While there are no established surgical guidelines in malignancyrelated enteric fistulas, the guidelines for management of enteric fistulas in Crohn's Disease indicate that those with signs and symptoms of local or systemic infection should receive antibiotics and surgical intervention. ${ }^{7}$ Conservative, non-operative management should only be considered in those patients at highest risk of post-operative morbidity and mortality. The preferred approach to management consists of fluid resuscitation and broad-spectrum antibiotics along 
with resection of the involved colonic segment and splenectomy. ${ }^{7}$ In our case, subtotal colectomy and splenectomy were thought to involve unacceptably high risk. Thus, the surgeons opted for a less morbid surgical approach with loop ileostomy and transverse colostomy in hopes that chemotherapy could be given in a timely manner. Our case demonstrates that, regardless of the surgical management approach, colosplenic fistulas in the setting of colorectal cancer have high morbidity and mortality, and more robust management guidelines are needed.

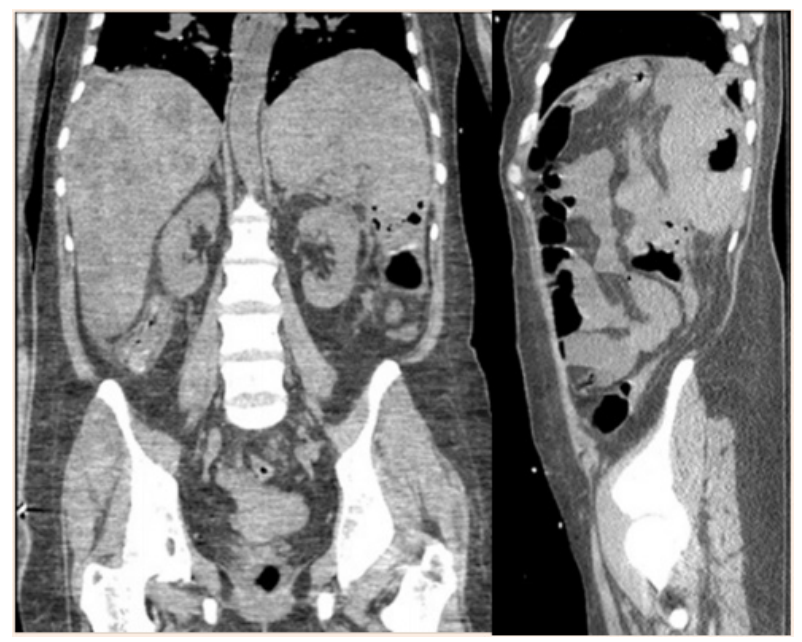

Figure 2 \& 3 CTAbdomen/Pelvis (coronal and sagittal) sections demonstrating splenic flexure wall thickening with normal bowel gas adjacent to splenic gas collection.

\section{Acknowledgements}

Amit P. Desai MD, David W. Wan MD.

\section{Conflicts of interest}

No financial interest or any conflict of interest exists.

\section{Patient consent form}

Patient deceased at time of case report writing and unable to provide consent.

\section{References}

1. Goldberg JB, Moses RA, Holubar SD. Colosplenic Fistula: a Highly Unusual Colonic Fistula. J Gastrointest Surg. 2012;16(12):2338-2340.

2. Hashemzadeh S, Pouryousef K, Oliaei Motlagh M, et al. Colosplenic fistula as a complication of splenic abscesses. ANZ J Surg. 2016;86(78):623.

3. Rowell DL, Longstreth GF. Colosplenic fistula and splenic abscess complicating Crohn's colitis. J Clin Gastroenterol. 1995;21(1):74-75.

4. Goldsmith PJ, Pine JK, Smith AM. An unusual case of rectal bleeding: colosplenic fistula complicating pancreatitis. Pancreas. 2011;40(2):316317.

5. Herreros de Tejada A, Giménez-Alvira L, Van den Brule E, et al. Severe splenic rupture after colorectal endoscopic submucosal dissection. World J Gastroenterol. 2014;20(28):9618-9620.

6. Hyun BH, Varga CF, Rubin RJ. Spontaneous and pathologic rupture of the spleen. Arch Surg. 1972;04(5):652-657.

7. Strong S, Steele SR, Boutrous M, et al. Clinical Practice Guideline for the Surgical Management of Crohn's Disease. Dis Colon Rectum. 2015;58(11):1021-1036. 